\title{
The effect of intravenous morphine compare to lidocaine on renal colic patients in emergency department
}

\author{
Alireza Esmaili ${ }^{1}$, Laleh Borjian ${ }^{1}$, Mohammadali Jafari ${ }^{1}$, Sedighe Vaziribozorg ${ }^{2}$ \\ 1 Department of Emergency, Shahid Sadoughi University of Medical Sciences, Yazd, Iran \\ 2 Department of Otolaryngology-Head and Neck Surgery, Otorhinolaryngology Research Center, Shahid Sadoughi University of \\ Medical Sciences, Yazd, Iran \\ *Corresponding Author: Dr. Laleh Borjian, Department of Emergency, Shahid Sadoughi University of Medical Sciences, Yazd, \\ Iran. \\ Email: lalehborjian2019@gmail.com; Tel: +9835381133372
}

Received: February 18, 2020; Accepted: March 31, 2020

\begin{abstract}
Introduction: The aim of this study was to compare the effect of intravenous morphine versus lidocaine on renal colic in patients admitted to the emergency department. Methods and Materials: In this clinical trial study, 80 patients with renal colic admitted to the emergency department were included. Patients were divided into two groups randomly, namely Lidocaine and morphine groups. Each patient's pain score was recorded 5 , 10 , 15 and 30 minutes after the injection using Numeric Pain Intensity Scale (NPIS). Results: There were significant decreases in mean of pain scores in both groups $(p<0.001)$. Pain reduction was significantly higher in Lidocaine-received group at 30th minutes after injection ( $<0.001)$. Having compared groups in terms of complications, it was found that only dizziness in patients who received Lidocaine was significantly higher than that of the other group $(p=0.016)$. Conclusion: Considering the significant reduction in pain intensity of all patients, the effect of both medications on the reduction of pain in renal colic is confirmed. Given that pain relief in Lidocaine group was significantly more than that in morphine group after 10 minutes of injection, it can be concluded that Lidocaine is more effective than morphine in controlling renal colic.
\end{abstract}

Keywords: Renal colic, Morphine, Lidocaine.

\section{INTRODUCTION}

Kidney and urinary tract stone are one of the common problems in the urinary tract which is associated with severe colic pain caused by obstruction, inflammation, and smooth muscle spasm. Depending on the location of the stone and obstruction, the pain may be felt in the flank or hypogastric regions radiating down into the testis or labia majora [1]. The classic manifestation of the disease is a sudden onset of pain radiating from the flank to the extremities, which is usually associated with microscopic hematuria (85\%), nausea, and vomiting. Costovertebral angle tenderness is also a common finding. Referral to the emergency department is common due to renal colic, and the rate of pain relapse in this disease is very high [2]. To relieve pain in these patients, various medications, including antiemetics, nonsteroidal anti-inflammatory drugs (NSAID), anti-diuretics, ketorolac, acetaminophen, etc. are introduced. One of the current treatment regimens in the renal colic is morphine sulfate (MS) [3]. One of the major problems of this medication is the risk of developing reliance [4], especially in addicts who enter the withdrawal period. As noted above, due to the high frequency of referral of these patients to the emergency department for receiving the medication, these individuals are very exposed to the MS-induced complications, especially its addiction. Lidocaine is an appropriate choice for treating visceral and central pains, and can be used wherever narcotics are inappropriate or cause side effects. Intravascular injection of Lidocaine is effective in controlling neuropathic pain such as pain induced by diabetic neuropathy, post-operative pain, and post herpetic neuralgia, headaches, and pain induced by neurological malignancies. Therefore, we decided to compare the analgesic effects of intravenous lidocaine and intravenous morphine in renal colic patients.

\section{MATERIALS AND METHODS}

After approval by the local ethics committee and obtaining written informed consent from all patients, in this double-blind 
clinical trial study 80 patients aged $18-55$ years old with renal colic involved. These patients referred to the emergency department with kidney pain (severe bilateral pain radiated down into the hypogastrium, testis, or labia majora), and their disease was confirmed by diagnostic sonography. After taking patients' medical histories, performing clinical examinations, and confirming the diagnosis of renal colic, the incidence of patients' pain was scored using numerical pain rating scale. The data were recorded using a pre-developed checklist. Other information, including patient's baseline blood pressure, history of kidney or heart failure, and pregnancy status was recorded on each patient's checklist. Patients with history of kidney failure, heart failure, drug addiction, and seizure as well as pregnant patients were excluded from the study. The patients were randomly assigned to the case $(A=$ intravenous Lidocaine) or control group ( $\mathrm{B}=$ intravenous morphine) using random number table. $1.5 \mathrm{mg} / \mathrm{kg}$ of intravenous Lidocaine (2\%) and $0.1 \mathrm{mg} / \mathrm{kg}$ of intravenous morphine were slowly injected to Group A and group $B$, respectively by a nurse. In addition, $100 \mathrm{ml}$ of normal saline was infused over 20 minutes to both groups. The patient and the prescriber both were blinded about the administered medication. During the infusion of the medication, the evaluator checked the patient for the drug-induced complications (i.e. nausea and vomiting, hypotension, light headedness, and dizziness). In case of severe complications, the infusion was discontinued, the researcher was informed, and the second analgesic medication, intravenous ketorolac, was administered. Patients' pain score was recorded at $5,10,15$, and 30 minutes following injection. Positive response to treatment was defined as the reduction of patient's pain for 3 scores or more, while negative response to treatment was defined as lack of patient's pain decrease for 3 scores within 30 minutes following medication administration. All the patients were monitored in the emergency department 1 hour after medication administration to find out any complications and to determine the relapse rate of symptoms. For data analysis, SPSS version 21 and appropriate statistical tests was used. P.value $<0.05$ considered significant.

\section{RESULTS}

In this study, 80 patients were enrolled who were randomly divided into two groups of intravenous morphine $(n=40)$ and intravenous Lidocaine $(n=40)$. The mean age of morphine group was $36.66 \pm 6.88$ years old, and that of Lidocaine group was $36.02 \pm 5.07$ years old, indicating no significant difference $(P=$ 0.632 ). During the study, both groups experienced significantly lower pain over time $(\mathrm{P}<0.001)$. In morphine group, the mean of patients' pain score reduced from $8.3 \pm 1.7$ (five minutes after administration) to $4.9 \pm 1.9$ (thirty minutes after administration). In Lidocaine group, the mean of patients' pain score also decreased from $8.2 \pm 1.6$ (five minutes after administration) to $2.6 \pm 2.1$ (thirty minutes after administration). Having compared two groups, it was detected that the amount of pain reduction in two groups was similar within 10 minutes after injection, and the difference between groups started after 10 minutes, in a way that the amount of pain reduction in Lidocaine group was significantly more than that of second group after 30 minutes $(P<0.001)$. With respect to drug-induced complications, only dizziness in patients who received Lidocaine was significantly higher than that of the other group $(P=0.016)$.

\section{DISCUSSION}

In this clinical trial study, the analgesic effect of intravenous Lidocaine was compared with that of intravenous morphine in patients suffering from renal colic. Based on the results, there was a significant pain reduction in patients following intravenous injection of both medications. In addition, pain reduction after 10 minutes was more remarkable in Lidocaine group; however, pain in Lidocaine group was significantly reduced 30 minutes after injection compared to pain reduction in morphine group. In a systematic review in 2017, the effect of intravenous Lidocaine on pain management was evaluated using 61 studies. Among the 6 randomized controlled trials included, intravenous lidocaine had efficacy equivalent to that of active controls (morphine and ketorolac) in 2 studies, and was better than active controls (morphine) in 2 other studies. In particular, intravenous lidocaine had pain score reduction comparable to or higher than that of intravenous morphine for pain associated with renal colic and critical limb ischemia [5]. Our findings also assured pain reduction efficacy of Lidocaine in patients with renal colic. In Firouzian et al.'s study (2016), the analgesic effect of Lidocaine in combination with morphine was compared with that of morphine in 110 patients referred to emergency department due to acute renal colic. In aforementioned study, no significant difference was discovered, though both medications were effective in reducing the intensity of pain. Additionally, analgesic effects were observed at a shorter time in patients received Lidocaine in combination with morphine. Drug-induced complications such as nausea and the need for anti-nausea treatment were less reported in Lidocaine and morphine group [6]. In contrast to our findings, no significant difference was detected between two groups. Motamed et al., (2017) studied 90 patients with colic pain in the flank comparing the analgesic effects of Lidocaine with fentanyl at 5, 10, 15, and 30 minutes after injection. They reported no significant difference between groups in terms of pain reduction [7], which is contrast with findings of current investigation. Vahidi et al., (2015) compared the efficacy of intravenous morphine and Lidocaine in management of pain in limb ischemia. In aforementioned study, 63 patients with limb ischemia were investigated. They indicated that intravenous Lidocaine offered greater pain relief within 15 to 30 minutes after the intervention [8]. In line with our findings, they also yielded higher analgesic effect of Lidocaine on pain. The analgesic effects of intravenous Lidocaine and intravenous morphine on patients with acute renal colic who referred to emergency department was done by Sulimanupour et al., (2012). In their study, 240 patients aged 18-65 years were evaluated in terms of management of pain induced by renal colic. Patients who received Lidocaine experienced significantly lower pain compared to those who received morphine. In addition, 11 patients in morphine-treated group experienced nausea and vomiting, but no one reported these complications in Lidocaine group [9]. The findings of aforementioned study are in line with ours indicating the higher efficacy of Lidocaine in deceasing pain caused by renal colic. In a recent study by $\mathrm{Al}$ Boye et al., (2014), it was found that addition of Lidocaine $1 \%$ to $20 \mathrm{mg}$ morphine could significantly reduce pain compared to mere administration of $20 \mathrm{mg}$ morphine. In their study, three groups (lidocaine $1 \%$ plus $20 \mathrm{mg}$ morphine for the first group, lidocaine $1 \%$ plus $10 \mathrm{mg}$ morphine for the second group, and only $20 \mathrm{mg}$ morphine for the third group) did not differ significantly in terms of drug-induced complications [10]. In other words, they concluded that the combined effects of two medications were more the effect of individual medication, which is in line with our findings revealing that Lidocaine had a significant effect on pain relief.

\section{CONCLUSION}

Considering the significant reduction in pain intensity of all patients, the effect of both medications on the reduction of pain in renal colic is confirmed based on our findings. Given that pain relief in Lidocaine group was significantly more than that in morphine group after 10 minutes of injection, it can be concluded 
that Lidocaine is more effective than morphine in controlling renal colic.

\section{Consent}

Informed consent was obtained from all participants of the study.

\section{Conflict of Interest}

We declare that we have no conflict of interest.

\section{Financial Support}

None declared.

\section{REFERENCES}

1. Marx J, Walls R, Hockberger R. Rosen's Emergency MedicineConcepts and Clinical Practice E-Book: Elsevier Health Sciences; 2013.

2. Moe OW. Kidney stones: pathophysiology and medical management. The lancet. 2006;367(9507):333-44.

3. Shaker SH, Mosadegh R, Jalali F, Zavareh M. Comparison of intravenous morphine and ketorolac in renal colic patients admitted to Firoozgar and Hazrat Rasoul e Akram hospitals. 2017.

4. Payandemehr P, Jalili M, Davani BM, Dehpour AR. Sublingual buprenorphine for acute renal colic pain management: a doubleblind, randomized controlled trial. International journal of emergency medicine. 2014;7(1):1.

5. e Silva LOJ, Scherber K, Cabrera D, Motov S, Erwin PJ, West CP, et al. Safety and efficacy of intravenous lidocaine for pain management in the emergency department: a systematic review. Annals of emergency medicine. 2018;72(2):135-44. e3.

6. Firouzian A, Alipour A, Dezfouli HR, Kiasari AZ, Baradari AG, Zeydi $A E$, et al. Does lidocaine as an adjuvant to morphine improve pain relief in patients presenting to the ED with acute renal colic? A double-blind, randomized controlled trial. The American journal of emergency medicine. 2016;34(3):443-8.

7. Motamed $\mathrm{H}$, Verki MM. Intravenous lidocaine compared to fentanyl in renal colic pain management; a randomized clinical trial. Emergency. 2017;5(1).

8. Vahidi E, Shakoor D, Meybodi MA, Saeedi M. Comparison of intravenous lidocaine versus morphine in alleviating pain in patients with critical limb ischaemia. Emerg Med J. 2015;32(7):516-9.

9. Soleimanpour H, Hassanzadeh K, Vaezi H, Golzari SE, Esfanjani RM, Soleimanpour M. Effectiveness of intravenous lidocaine versus intravenous morphine for patients with renal colic in the emergency department. BMC urology. 2012;12(1):13.

10. Alebouyeh MR, Imani F, Rahimzadeh P, Entezary SR, Faiz SHR, Soraya P. Analgesic effects of adding lidocaine to morphine pumps after orthopedic surgeries. Journal of research in medical sciences: the official journal of Isfahan University of Medical Sciences. 2014;19(2):122. 